\title{
The prognostic nomogram in platinum-resistant ovarian cancer: how to develop and validate?
}

\author{
Kuo-Chang Wen ${ }^{1,2}$, Pi-Lin Sung ${ }^{1,2}$, Hung-Cheng Lai ${ }^{1,2,3,4}$ \\ ${ }^{1}$ Department of Obstetrics and Gynecology, Shuang Ho Hospital, New Taipei City; ${ }^{2}$ Department of Obstetrics and Gynecology, School of Medicine, \\ College of Medicine, Taipei Medical University, Taipei; ${ }^{3}$ Translational Epigenetic Center, Shuang Ho Hospital, Taipei Medical University, New \\ Taipei City; ${ }^{4}$ Department and Graduate Institute of Biochemistry, National Defense Medical Center, Taipei \\ Correspondence to: Hung-Cheng Lai. Department of Obstetrics and Gynecology, Shuang Ho Hospital, New Taipei City. \\ Email: hclai30656@gmail.com; hclai@s.tmu.edu.tw. \\ Comment on: Lee CK, Asher R, Friedlander M, et al. Development and validation of a prognostic nomogram for overall survival in patients with \\ platinum-resistant ovarian cancer treated with chemotherapy. Eur J Cancer 2019;117:99-106.
}

Submitted Jan 20, 2020. Accepted for publication Jun 15, 2020.

doi: $10.21037 / \mathrm{cco}-2020-002$

View this article at: http://dx.doi.org/10.21037/cco-2020-002

Dr. Lee et al. reported "Development and validation of a prognostic nomogram for overall survival in patients with platinum-resistant ovarian cancer treated with chemotherapy", on Eur J Cancer (1). Via the data from chemotherapy-only arm in AURELIA and randomized cases in CARTAXHY trial as training cohort, Dr. Lee created a nomogram to predict the prognosis in ovarian cancer. This prognostic nomogram was developed and generated by the pretreatment characteristics such as patients' performance, tumor size, or CA-125, etc., under multivariable proportional hazards models. Further, Dr. Lee applied their nomogram in other two large-scale data, bevacizumab-chemotherapy arm of AURELIA and PENELOPE trial as validation cohort, to calibrate the performance. They selected six significant predicting markers including patients' performance, intra-abdominal ascites, size of largest tumor, serum CA-125, platinum-free interval, and platinum-resistance status. Surprisingly, we can notice rather powerful stratification of overall survival (OS) in the patients with platinum-resistant ovarian cancer. Not only training cohort but also validation cohort, Dr. Lee's nomogram can discriminately analyze OS in different prognosis groups. In training cohort (chemotherapy-only arm in AURELIA and randomized cases in CARTAXHY), the median OS of good, intermediate, and poor prognosis groups were 25.3, 15.2, and 7.4 months, respectively. As to validation cohort, PENELOPE (C-statistic 0.59) and bevacizumab-chemotherapy arm of AURELI (C-statistic 0.67), the median OS were 18.5, 10.3, 5.8 months and
26.7, 13.8, 10.0 months in the good, intermediate and poor prognosis groups. Their conclusion revealed that their nomogram may effectively predict or counsel the prognosis and survival status for the patients of platinum-resistant ovarian cancer. The current study is interesting and worthy of further discussion.

Firstly, these authors mainly developed several nomograms for different subtypes of ovarian cancer and other cancer, such as progression-free survival and overall survival in platinum-sensitive recurrent ovarian cancer (2), advanced breast cancer (3), metastatic gastrointestinal stromal tumor treated with imatinib (4). Therefore, they had good experiences to create a prediction tool. Nomogram, or called nomograph, is a graphical and mathematical calculating method to create a diagram reaching estimated graphical computation. A set of $\mathrm{n}$ scales, and one for each variable in an equation, were included in the nomogram. Knowing the values of $n-1$ variables, the value of the unknown variable can be found; alternatively, the relationship between the unfixed ones can be studied by fixing the values of some variables. It is widely used in chemistry, aeronautics, astronomical calculations, and oncology, etc. In the field of oncology, it is frequently used to predict cancer survival mainly because of reducing statistical predictive models into a single numerical estimate of the probability of an event, like patients' alive status (overall survival) and tumor recurrence (progression-free survival). The generation of these estimates by user-friendly graphical 
interfaces facilitates the use of nomograms in clinical decision making. In practice, the clinicians can construct a new nomogram to aid in the prognosis of individual cases by using the clinical information and data from published datasets. After comparison with original cohort, interesting predicting parameters may be identified and further validated in current existed nomogram. By this model, other specialists had also designed and constructed a nomogram, six-mRNA risk score system, to predict the clinical prognosis and platinum response in ovarian cancer (5). Dr. Wang et al. downloaded copy number variation (CNV) information from The Cancer Genome Atlas (TCGA) and further validated with GSE63885 datasets. The panel combined with six mRNA genes (TBXT, SYNM, TEKT5, $G D F 3, S L C 22 A 3$, and $C A C N A 1 C$ ) was found to be an independent factor in prognosis.

Furthermore, Dr. Lee and colleagues applied six significant OS predictors, including patients' performance, intra-abdominal ascites, size of largest tumor, serum CA125, platinum-free interval, and platinum-resistance status, in their paper. These six parameters all were proven to be the important prognostic factor in human ovarian cancer according to many published studies. Performance status is an evaluation to score daily well-being and life activity of cancer patients. Eastern Cooperative Oncology Group (ECOG) (6) or Karnofsky system (7) are mostly used to rank performance status. Not only before treatment but also 3 months after chemotherapy, it can both be a significant predictor in overall survival (8). Ascites may be found in more half ovarian cancer patients; malignant ascites were associated to peritoneal seeding or poor prognosis (9). Malignant cells in ascites or peritoneal washing can be defined as IC3 in FIGO staging system. Ascites derived tumor cells were even highlighted as the cancer source carrying stem-like capability. As to the size of largest tumor, the average size in late stage was significantly smaller than that in early stage (4.8vs. $10.7 \mathrm{~cm}$, respectively) (10). The median survival months in the cases with ovarian tumor less than or equal to $6 \mathrm{~cm}$ was 17 months, while the median survival of the cases with tumor greater than $6 \mathrm{~cm}$ was 36 months $(\mathrm{P}=0.003)$ (11). The authors using prognostic nomogram CA-125 was also investigated in Dr. Matte and colleagues' paper (12). They found that serum CA125/ascites leptin being a potential novel biomarker to predict baseline clinical drug resistance to first-line chemotherapy and a predictor for poor outcome in ovarian cancer patients. Serum CA125/ascites leptin ratio was showed to be significantly elevated in platinum resistant, compared to drug-sensitive diseases. "Platinum resistant", was historically defined as ovarian cancer recurrence or tumor progression within 6 months after or during first-line adjuvant platinum-based chemotherapy. The platinum sensitivity and platinum-free interval were both the most important factors to predict the response of first line chemotherapy and overall survival (13). From many preclinical and clinical studies, the extension of platinumfree interval by using non-platinum-based regimen, such as Lipo-Dox or Topotecan, may restore platinum sensitive status, thus allowing improving survival (14). Except the nomogram factors selected in this paper, other parameters including serum HE4 (15), NLR/PLR (16), or molecular profiles, proven as novel indicators, may also be selected to generate new nomogram for chemo-resistance and prognosis in EOC (epithelial ovarian cancer).

On the third, nomogram is usually as a prognostic device to predict prognosis (17). However, how to simplify the complex mathematical formula is the key to improve nomogram friendly. A "Cancer-Specific Survival Following Surgery" provided by Memorial Sloan Kettering Cancer Center (www.mskcc.org/nomograms/ovarian) (18) is to predict the 5 -year survival of ovarian cancer after surgery. After finishing the worksheet including age, stage, albumin level, and maximal diameter of tumor, ASA score, and family history of hereditary breast/ovary cancer, the cancer specific survival can be calculated. Therefore, the strength of the article is that the establishment of this kind of nomogram can be easily converted into an online tool for other physicians. The online tool can provide a convenient and effective evaluation method for other physicians. Other clinician can apply a published nomogram that was generated using data on cohorts that might be different than one's own population of interest. The training or validated groups for this nomogram have their own unique personal or disease characteristics. It generates that nomogram may not be able to be effectively represented in another group of patients. Under the circumstances, Iasonos et al. (19) provided several reviewing points for clinicians to perform their assessment to apply an existing nomogram for their patients, including such as data population, clinical outcome, all variables under considerations, reporting system and the validation of this nomogram. They also identify a set of basic questions for clinicians to consider before applying a nomogram in the clinical setting and let clinicians to evaluate accessibility of this nomogram based on their own patients characteristics, clinical characteristics (tumor type), previous treatment, race/ethnicity, previous 
therapy, or clinical size and others factors. By their methodological approach, the researchers may be able to reduce statistical operation in using or even generating a nomogram.

Finally, although the nomogram is a powerful tool in prediction of survival, we must also remind some important issues in EOC. There are several different histological subtypes, such as high/low grade serous, clear cell, endometrioid, accompanied with their salient features. In HGSOC (high grade serous ovarian cancer), the key target genes are TP53, BRCA1/2, homologous recombination repair $(H R R)$ genes; different genes like $B R A F, K R A S$, or PI3KCA, are more mutated in LGSOC (low grade serous ovarian cancer) (20). If we generate one predictive nomogram in HGSOC, it is unknown whether this can also be fitted in LGSOC. In the future, as the authors mentioned, the nomogram can be developed with big data from precision medicine, such as genotypes, epigenetic study and even omics studies, in order to get a more stable and wildly used prediction nomogram system.

\section{Acknowledgments}

Funding: None.

\section{Footnote}

Provenance and Peer Review: This article was commissioned by the editorial office, Chinese Clinical Oncology. The article did not undergo external peer review.

Conflicts of Interest: All authors have completed the ICMJE uniform disclosure form (available at http://dx.doi. org/10.21037/cco-2020-002). The authors have no conflicts of interest to declare.

Ethical Statement: The authors are accountable for all aspects of the work in ensuring that questions related to the accuracy or integrity of any part of the work are appropriately investigated and resolved.

Open Access Statement: This is an Open Access article distributed in accordance with the Creative Commons Attribution-NonCommercial-NoDerivs 4.0 International License (CC BY-NC-ND 4.0), which permits the noncommercial replication and distribution of the article with the strict proviso that no changes or edits are made and the original work is properly cited (including links to both the formal publication through the relevant DOI and the license). See: https://creativecommons.org/licenses/by-nc-nd/4.0/.

\section{References}

1. Lee CK, Asher R, Friedlander M, et al. Development and validation of a prognostic nomogram for overall survival in patients with platinum-resistant ovarian cancer treated with chemotherapy. Eur J Cancer 2019;117:99-106.

2. Lee CK, Simes RJ, Brown C, et al. A prognostic nomogram to predict overall survival in patients with platinum-sensitive recurrent ovarian cancer. Ann Oncol 2013;24:937-43.

3. Lee CK, Hudson M, Stockler M, et al. A nomogram to predict survival time in women starting first-line chemotherapy for advanced breast cancer. Breast Cancer Res Treat 2011;129:467-76.

4. Lee CK, Goldstein D, Gibbs E, et al. Development and validation of prognostic nomograms for metastatic gastrointestinal stromal tumour treated with imatinib. Eur J Cancer 2015;51:852-60.

5. Wang Q, Lu Z, Ma J, et al. Six-mRNA risk score system and nomogram constructed for patients with ovarian cancer. Oncol Lett 2019;18:1235-45.

6. Oken MM, Creech RH, Tormey DC, et al. Toxicity and response criteria of the Eastern Cooperative Oncology Group. Am J Clin Oncol 1982;5:649-55.

7. Karnofsky DA AW, Craver LF, et al. The Use of the Nitrogen Mustards in the Palliative Treatment of Carcinoma - with Particular Reference to Bronchogenic Carcinoma. Cancer 1948:634-56.

8. Carey MS, Bacon M, Tu D, et al. The prognostic effects of performance status and quality of life scores on progression-free survival and overall survival in advanced ovarian cancer. Gynecol Oncol 2008;108:100-5.

9. Ahmed N, Stenvers KL. Getting to know ovarian cancer ascites: opportunities for targeted therapy-based translational research. Front Oncol 2013;3:256.

10. Horvath LE WT, Boucher K, et al. The relationship between tumor size and stage in early versus advanced ovarian cancer. Med Hypotheses 2013:684-7.

11. Stratton JF, Pharoah P, Tidy JA, et al. An analysis of ovarian tumor diameter and survival. Int J Gynecol Cancer 2000;10:449-51.

12. Matte I, Garde-Granger P, Bessette P, et al. Serum CA125 and ascites leptin level ratio predicts baseline clinical resistance to first-line platinum-based treatment and poor prognosis in patients with high grade serous ovarian 
cancer. Am J Cancer Res 2019;9:160-70.

13. Davis A, Tinker AV, Friedlander M. "Platinum resistant" ovarian cancer: what is it, who to treat and how to measure benefit? Gynecol Oncol 2014;133:624-31.

14. Tomao F, D'Incalci M, Biagioli E, et al. Restoring platinum sensitivity in recurrent ovarian cancer by extending the platinum-free interval: Myth or reality? Cancer 2017;123:3450-9.

15. Vallius T, Hynninen J, Auranen A, et al. Postoperative human epididymis protein 4 predicts primary therapy outcome in advanced epithelial ovarian cancer. Tumour Biol 2017;39:1010428317691189.

16. Miao Y, Yan Q, Li S, et al. Neutrophil to lymphocyte ratio and platelet to lymphocyte ratio are predictive of

Cite this article as: Wen KC, Sung PL, Lai HC. The prognostic nomogram in platinum-resistant ovarian cancer: how to develop and validate? Chin Clin Oncol 2021;10(3):31. doi: 10.21037/cco-2020-002 chemotherapeutic response and prognosis in epithelial ovarian cancer patients treated with platinum-based chemotherapy. Cancer Biomark 2016;17:33-40.

17. Balachandran VP, Gonen M, Smith JJ, et al. Nomograms in oncology: more than meets the eye. Lancet Oncol 2015;16:e173-80.

18. Barlin JN, Yu C, Hill EK, et al. Nomogram for predicting 5 -year disease-specific mortality after primary surgery for epithelial ovarian cancer. Gynecol Oncol 2012;125:25-30.

19. Iasonos A, Schrag D, Raj GV, et al. How to build and interpret a nomogram for cancer prognosis. J Clin Oncol 2008;26:1364-70.

20. Lheureux S, Gourley C, Vergote I, et al. Epithelial ovarian cancer. Lancet 2019;393:1240-53. 